\title{
Effects of interpolated items on the association effect in lexical decision tasks
}

\author{
EILEEN DAVELAAR and MAX COLTHEART \\ Reading University, Reading, England RG6 2AL
}

\begin{abstract}
Meyer, Schvaneveldt, and Ruddy (1972) reported that in a lexical decision task, the word association effect was maintained when two associated words were separated by an unassociated word but was eliminated when the two were separated by a nonword. They suggest that the effect is eliminated in the latter case because a negative decision may result in a "resetting" of the memory system to a neutral level. The present investigation comprised a partial replication of the above study. Associated words were separated either by unassociated words, pronounceable nonwords, or unpronounceable nonwords. Results indicated that the word association effect was significant regardless of whether the intervening item was a word or a nonword; and, in addition, the pronounceability of the nonword was not found to influence the magnitude of the effect. The data were taken to provide further support for the spreading excitation model.
\end{abstract}

The word association effect in lexical decision tasks has been well documented in recent years by Meyer and his colleagues (Meyer \& Schvaneveldt, 1971; Meyer, Schvaneveldt, \& Ruddy, 1975; Meyer, Schvaneveldt \& Ruddy, Note 1). Briefly, these researchers have found that subjects require significantly less time to decide that a given letter string (e.g., BUTTER) is a word if that letter string is preceded by an associated word (e.g., BREAD) than if it is preceded by an unassociated word (e.g., NURSE). This finding has been consistent whether the associated words were presented simultaneously, successively in pairs, or successively with an intervening unassociated word.

Meyer et al. have attempted to account for these findings in terms of a spreading excitation model. This model proposes that, as a subject processes a particular word, excitation occurs in that word's neural pathways. This neural excitation, these authors suggest, is not restricted solely to the pathways of the word in question but spreads to the pathways of other words with which it is associated. This spreading excitation consequently reduces the recognition thresholds for associated items since some level of excitation is already present in the appropriate pathways.

With one exception, this model appears to account satisfactorily for their findings. The exception occurs in their three-string successive presentation experiment (Meyer, Schvaneveldt, \& Ruddy, Note 1). The results from this study indicated that while a significant word association effect remained when two associated words were separated by an unassociated word, the effect was eliminated when the two associates were separated by a nonword. In the same paper, the authors demonstrated that a trace decay notion could not explain the elimination of the effect. Consequently, the spreading excitation model cannot account for this finding without some revision.

The revision offered by Meyer et al. is not entirely satisfactory. They suggest, somewhat vaguely, that the processing of a nonword somehow "resets" the memory system to a neutral level, resulting in the elimination of any excitation previously present in the system. While this explanation may be correct, it is not obvious why a negative decision should have such a dramatic effect on existing excitation levels while positive decisions have no effect.

One possible and perhaps more parsimonious explanation may lie in the nature of the stimuli employed in the associated conditions. Since the authors make no mention of associative strength parameters, it may be that in the condition where the middle item was was a nonword, more weakly associated pairs were used than in the condition where the middle item was an unassociated word. The weakness of the associates per se may have resulted in an insignificant effect. The present study was undertaken in part to investigate this possibility.

A further aim of the present experiment was to observe the effects of varying the nature of the intervening nonwords. Since Meyer et al. employed only pronounceable nonwords, it was thought advisable to attempt to replicate their study with the use of unpronounceable nonwords as well as with pronounceable ones, while controlling for associatve strength across all conditions. If the "no" response to an unpronounceable nonword is made by detecting illegality prior to lexical access (Rubenstein, Lewis, \& Rubenstein, 1971), any "resetting" due to an unsuccessful lexical search would not occur in this case; it would only occur with pronounceable nonwords, whose processing does require lexical search. On the other hand, if Meyer et al. are correct and any negative 
decision does indeed "reset" the memory system to a neutral level, then the nature of the intervening nonword should have no differential effect with respect to the elimination of existing excitation levels.

\section{METHOD}

\section{Subjects}

A total of 30 subjects participated in the experiment. Half of these served in the pronounceable nonword condition and half in the unpronounceable nonword condition. The subjects were tested individually in single sessions lasting approximately $20 \mathrm{~min}$. All subjects were either graduate or undergraduate students at Reading University.

\section{Procedure}

The subjects were told that they were going to see isolated letter strings, some of which would be English words and some of which would be nonwords. Each was asked to press a yes key with his preferred hand if the string was a word and a no key with his nonpreferred hand if the string was a nonword. They were further instructed to respond as quickly and accurately as possible.

The letter strings were displayed on a remote scope connected to a PDP-12 computer, at a viewing distance of $40 \mathrm{in}$. The computer recorded whether the response was correct and the time elapsed between stimulus onset and subject's keypress. Each stimulus was erased as soon as a keypress occurred, followed by another stimulus $.5 \mathrm{sec}$ later. The sessions commenced with 15 practice trials (seven words, eight nonwords) in order to familiarize each subject with the task and the equipment.

\section{Materials}

The test stimuli consisted of 200 English words and 100 pronounceable or 100 unpronounceable nonwords, all between three and seven letters in length. Subjects saw either all the words and 100 pronounceable nonwords or all the words and 100 unpronounceable nonwords, for a total of 300 test trials. The 200 words were comprised of 40 pairs of associates selected from Miller (1970) and 120 unassociated words. The associated pairs were divided such that 20 pairs were assigned to the AUA condition and 20 pairs to the ANA condition. Furthermore, they were assigned to these conditions such that there was no difference between the two with respect to associative strength (Condition AUA, mean $=157$, range $=87-311$; Condition ANA mean $=153$, range $=88-281$ ). Associative strength was measured simply in terms of the frequency of the given response to an item as indicated by Miller (1970) for the English sample $(\mathrm{N}=400)$.

The 120 unassociated words were selected as follows: first, 20 common words were chosen to serve as the midline item in the AUA condition. Since this condition was to be compared with the UUU condition, the three mutually unrelated words in the latter condition were selected such that they were of the same word frequency as their matching items in the AUA condition for each trial. For example, the AUA condition contained the trial sequence DARK-EVEN-LIGHT. The matching sequence in the UUU condition was EAST-MOST-EVER. These three particular items were employed because DARK and EAST share the same word frequency, as do EVEN and MOST and LIGHT and EVER. Additional efforts were made to equate these items for syntactical category and number of letters and syllables. An identical procedure was used to equate the $A$ and $U$ items in the ANA and UNU conditions.

The 100 nonwords were constructed, in the case where they were pronounceable, from common words by changing one or occasionally two letters while maintaining pronounceability. In the case where they were unpronounceable, these same nonwords were altered such that all the vowels were replaced by consonants. Forty of these items served as the nonwords in the ANA and UNU conditions, while the remaining 60 items acted as fillers randomly dispersed between test trials. A complete list of the items in their respective conditions appears in Appendix A.

\section{Design}

The experimental design comprised a partial replication of the Meyer et al. (Meyer, Schvaneveldt, \& Ruddy, Note 1) three-string successive presentation study. Only four of their conditions were employed. These were the AUA condition, in which two associated words were spearated by an unassociated word; the ANA condition, in which two successive associated words were separated by a nonword; the UUU condition, consisting of three successive unassociated words; and the UNU condition, in which two successive unassociated words were separated by a nonword. The trials in all four conditions were randomized, with each subject receiving all conditions. The word items remained the same for all subjects in all conditions while the nonword items were substituted such that half the subjects received pronounceable nonwords throughout and half received unpronounceable nonwords throughout. Only the response times to the third items in each condition served as data for subsequent analysis.

\section{RESULTS}

The mean response times of each subject in each condition served as the data for analysis. Several criteria were applied to the data to obtain these means. First, any reaction time more than $2 \mathrm{SD}$ away from the mean for that condition was eliminated. Where this occurred, the matched trial in the comparison condition was also eliminated. Secondly, where an error occurred on the third item of a trial, that trial along with its matched trial in the comparison condition was eliminated. Finally, if an error occurred on the middle item of a trial, that trial and its matched trial in the comparison condition were also eliminated. This last procedure was felt to be necessary since an error on a middle item would change the response sequence across comparison trials. These criteria resulted in $12 \%$ of the total trials being eliminated from further analysis, the majority (8\%) due to errors on the middle items.

The resulting data were analyzed according to the procedure outlined by Clark (1973). The means for subjects, shown in Table 1, were submitted to a split plot analysis of variance design with pronounceable vs. unpronounceable nonwords as a between factor and both association vs. unassociation and word vs. nonword middle item as within factors. Similarly, the means for words, also shown in Table 1, were submitted to a split plot analysis of variance design with word vs. nonword middle item as a between factor and both association vs. unassociation and pronounceable vs. unpronounceable nonwords as within factors.

The analysis showed significant effects of both association, $F^{\prime} \min (1 / 65.95)=12.08, \mathrm{p}<.01$ and middle item $F^{\prime} \min (1 / 64.69)=5.15, p<.05$. Since none of the remaining $F^{\prime}$ mins were comprised of even one significant simple $F$, none of these could have reached significance at their $F^{\prime} \max$ values (Clark, 1973, p. 356). The 
significant effect of the association factor indicates that the word association effect was obtained in both the AUA and ANA conditions. Furthermore, since the association factor did not significantly interact with any of the other factors present, the results demonstrate that the word association effect is not influenced either by the word-nonword nature of the intervening item or by the pronounceability of that item. The significant effect of the word vs. nonword middle item indicates that the AUA and UUU conditions were faster than the ANA and UNU conditions. This result is almost certainly due to the fact that the former two conditions required a "yes-yes-yes" response sequence with the same hand whereas the latter two conditions required a "yes-no-yes" response sequence with a change in hands for the "no" response.

\section{DISCUSSION}

The main finding of the experiment is that the word association effect was maintained in the three-string successive presentation procedure regardless of the nature of the letter
Table 1

Mean RTs (Milliseconds) for Subjects and Words Across All Conditions

\begin{tabular}{llrrrr}
\hline & & AUA & UUU & ANA & UNU \\
\hline Pronounceable & Subjects & 508 & 544 & 536 & 584 \\
Nonwords & Words & 499 & 542 & 535 & 586 \\
Unpronounceable & Subjects & 486 & 505 & 497 & 519 \\
Nonwords & Words & 492 & 512 & 503 & 528 \\
\hline
\end{tabular}

string separating two associated words. In the case where the two associates were separated by an unassociated word, this result is in agreement with that reported by Meyer et al. (Note 1). In the case where the two associates were separated by a nonword, however, this result is contrary to that obtained by these authors. Indeed, since the association factor did not interact with either the word vs. nonword middle item factor or with the pronounceability vs. unpronounceability factor, it may be concluded that the magnitude of the word association effect was uninfluenced by either of these manipulations. Clearly then, the postulation of a "resetting" of the memory system to a neutral level following a negative decision, as suggested by Meyer et al., is no longer necessary; and the spreading excitation model can easily account for these data.

\section{APPENDIX A: STIMULI EMPLOYED IN EACH CONDITION}

Condition AUA: 1. dark-even-light, 2. table-glass-chair, 3. girl-house-boy, 4. high-whole-low, 5. bread-baby-butter, 6. hard-west-soft, 7. spider-child-web, 8. dream-talk-sleep, 9. green-small-grass, 10. command-whistle-order, 11. cold-loud-hot, 12. slow-warm-fast, 13. mutton-doctor-chop, 14. priest-cats-church, 15. soldier-kittens-sailor, 16. carpet-mother-floor, 17. blue-sound-sky, 18. thirsty-truck-drink, 19. lion-cars-tiger, 20. moon-stove-stars.

Condition UUU: 1. east-most-ever, 2. peace-clay-block, 3. land-three-street, 4. once-kind-dead, 5. seed-duty-victim, 6. strong-court-vast, 7. comet-class-ant, 8. grow-hear-join, 9. poor-few-chest, 10. allow-chisel-present, 11. heart-slim-wrong, 12. pale-flat-gray, 13. pillow-cattle-ape, 14. mare-figs-mind, 15. concert-pillars-manor, 16. suburb-music-wall, 17. bad-north-leg, 18. smoky-wage-youth, 19. chaos-parts-slogan, 20. goal-trick-scenes.

Condition ANA: 1. black-plake*(plnkt)**-white, 2. bitter-surn(smm)-sweet, 3. long-dael(dsml)-short, 4. king-nolp(ntlp)-queen, 5. rough-mocket(mfckpt)-smooth, 6. give-fibe(frbg)-take, 7. stem-lorse(lprst)-flower, 8. bath-slerge(slbrgt)-water, 9. fruit-sela(sflp)-apple, 10. hammer-plame(plbmc)-nail, 11. ocean-noom(nkbm)-seas, 12. afraid-meam(mpzm)-fear, 13. salt-frent(frbnt)-pepper, 14. needle-blone(blmnt)-thread, 15. squaredolk(dwlk)-round, 16. deep-wint(wbnt)-shallow, 17. eagle-pamb(plmb)-bird, 18. hungry-glat(glrt)-food, 19. hand-kile(ktlp)-foot, 20. city-mald(mgld)-town.

Condition UNU: 1. near-froup*(frnbp)**-best, 2. correct-fatch(fastch)-clean, 3. good-sorm(sprm)-clear, 4. pain-milt(mslt)-grace, 5. rare-kife(kgfd)-prime, 6. knew-sint(spnt)-come, 7. barn-deeth(dlbth)-victor, 8. card-sath(spth)-public, 9. crime-bola(btls)-gravel, 10. picket-cheem(chbgm)-twin, 11. pencil-nout(nlpt)-bags, 12. alive-borse(btrsf)-farm, 13. wave-firl(fnrl)-tower, 14. anchor-bront(brpnt)-client, 15. fine-mife(mlfw)-fresh, 16. firm-bame(blmv)-superb, 17. altar-pota(pktr)-horn, 18. noble-dest(dlst)-club, 19. head-sarp(strp)-dust, 20. room-lide(ledm)-plan.

* Nonwords employed in pronounceable condition.

$* *$ Nonwords employed in unpronounceable condition.

\section{REFERENCE NOTE}

1. Meyer, D. E., Schvaneveldt, R. W., \& Ruddy, M. G. Activation of lexical memory. Paper presented at the meeting of the Psychonomic Society, St. Louis, Missouri, November 1972.

\section{REFERENCES}

Clark, H. H. The language-as-fixed-effect-fallacy: A critique of language statistics in psychological research. Journal of Verbal Learning and Verbal Behaviour, 1973, 12, 335-359. 
Miller, K. M. Free-association responses of English and Australian students to 100 words from the Kent-Rasanoff word association test. In L. Postman \& G. Keppel (Eds.), Norms of nord association. New York: Academic Press, 1970.

MeYer, D. E.. \& Schaveneldt, R. W. Facilitation in recognizing pairs of words: Evidence of a dependence between retrieval operations. Journal of Experimental Psychology, 1971, 90. 227-234.

Meyer, D. E., Schvaneveldt, R. W., \& Ruddy, M. G. Loci of contextual effects on visual word recognition. In P. M. A. Rabbitt \& S. Dornic (Eds.), Attention and performance (Vol. V). New York: Academic Press, 1975.
Rubenstein, H., Lewis, S. S., \& Rubenstein, M. A. Evidence for phonemic recoding in visual word recognition. Journal of Verbal Learning and Verbal Behaviour. 1971, 10. 657-675.

(Received for publication June 9, 1975.) 